\title{
Automorphisms of Algebras and Bochner's Property for Vector Orthogonal Polynomials
}

\author{
Emil HOROZOV ${ }^{\dagger \ddagger}$
}

$\dagger$ Department of Mathematics and Informatics, Sofia University, 5 J. Bourchier Blvd., Sofia 1126, Bulgaria

$\dagger$ Institute of Mathematics and Informatics, Bulg. Acad. of Sci., Acad. G. Bonchev Str., Block 8, 1113 Sofia, Bulgaria

E-mail: horozov@fmi.uni-sofia.bg

Received January 26, 2016, in final form May 12, 2016; Published online May 19, 2016 http://dx.doi.org/10.3842/SIGMA.2016.050

\begin{abstract}
We construct new families of vector orthogonal polynomials that have the property to be eigenfunctions of some differential operator. They are extensions of the Hermite and Laguerre polynomial systems. A third family, whose first member has been found by Y. Ben Cheikh and K. Douak is also constructed. The ideas behind our approach lie in the studies of bispectral operators. We exploit automorphisms of associative algebras which transform elementary vector orthogonal polynomial systems which are eigenfunctions of a differential operator into other systems of this type.
\end{abstract}

Key words: vector orthogonal polynomials; finite recurrence relations; bispectral problem; Bochner theorem

2010 Mathematics Subject Classification: 34L20; 30C15; 33E05

\section{Introduction}

Salomon Bochner [11] has classified all systems of orthogonal polynomials $P_{n}(x), n=0,1, \ldots$ (with respect to some measure on the real line) that are also eigenfunctions of a second-order differential operator

$$
L\left(x, \partial_{x}\right)=A(x) \partial_{x}^{2}+B(x) \partial_{x}+C(x)
$$

with eigenvalues $\lambda(n)$. Here the coefficients $A, B, C$ of the differential equation do not depend on the index (degree) $n$ of the polynomial $P_{n}(x)$. The orthogonality condition, due to a classical theorem by Favard-Shohat is equivalent to the well known 3-terms recursion relation

$$
x P_{n}=P_{n+1}+\beta(n) P_{n}+\gamma(n) P_{n-1},
$$

where $\beta(n), \gamma(n)>0$ are constants, depending on $n .{ }^{1}$ Bochner's theorem states that all polynomial systems with such properties are the classical orthogonal polynomials of Hermite, Laguerre and Jacobi. From now on we will replace the condition of orthogonality with respect to a measure with orthogonality with respect to a non-degenerate functional on the space of the polynomials of one variable $\mathbb{C}[x]$ with complex coefficients. In this setting we have to add to the classical orthogonal polynomials also the Bessel polynomials.

Generalizations of Bochner's result were suggested long ago by H.L. Krall [29]. He classified all order 4 differential operators which have a family of orthogonal polynomials as eigenfunctions.

\footnotetext{
*This paper is a contribution to the Special Issue on Orthogonal Polynomials, Special Functions and Applications. The full collection is available at http://www.emis.de/journals/SIGMA/OPSFA2015.html

${ }^{1}$ Here we use polynomials, normalized by the condition that the coefficient of their highest-order term is 1 .
} 
Later many contributions in this direction were made by T. Koornwinder [28], L. Littlejohn [30], J. Koekoek, R. Koekoek [27], etc.

In recent times there is much activity in generalizations and versions of the classical result of Bochner, see, e.g., [17, 18, 20, 24, 25]. An important role in some of these generalizations is played by ideas from the study of the bispectral problem, initiated in [16]. Even translating the results of Bochner and Krall into this language already gives a good basis to continue investigations [20]. It goes as follows. Let us introduce the function $\psi(x, n)=P_{n}(x)$. If we write the right-hand side of the 3 -term recursion relation as a difference operator $\Lambda(n)$ acting on functions $f(n)$ of the discrete variable $n$ as

$$
\Lambda(n) f(n)=f(n+1)+\beta(n) f(n)+\gamma(n) f(n-1),
$$

then the 3 -term recursion relation can be written as

$$
x \psi(x, n)=\Lambda(n) \psi(x, n) .
$$

This means that $\psi(x, n)$ is an eigenfunction of the discrete operator $\Lambda$ with eigenvalue $x$ and of a differential operator $L\left(x, \partial_{x}\right)$ with eigenvalue $\lambda_{n}$. Hence we can formulate the Bochner-Krall problem as

Find all systems of polynomials $P_{n}(x)$ that are eigenfunctions of a second-order difference operator $\Lambda(n)(1.2)$, where $\gamma(n) \neq 0$, with eigenvalue $x$ and of a differential operator $L\left(x, \partial_{x}\right)(1.1)$ with eigenvalues $\lambda(n)$.

In $[21,22]$ and several other papers the authors make use of Darboux transformations to construct families of systems of orthogonal polynomials, that are eigenfunctions of even-order differential operators. Some properties of these polynomial systems are different from the properties of the classical orthogonal polynomials. For example P. Iliev [24, 25] has studied the algebras of operators that have Bochner-Krall polynomials as eigenfunctions. These algebras have two or more generators, whereas the algebras corresponding to the classical OP are isomorphic to the algebra $\mathbb{C}[X]$.

Here we also use ideas found in the studies of bispectral operators but of different nature. Before explaining these as well as the main results let us introduce one more concept which is central for the present paper. This is the notion of vector orthogonal polynomials (VOP), introduced by J. van Iseghem [35]. Let $\left\{P_{n}(x)\right\}$ be a family of monic polynomials such that $\operatorname{deg} P_{n}=n$. Assume that they satisfy a $(d+2)$-term recursion relation, $d \geq 1$

$$
x P_{n}(x)=P_{n+1}+\sum_{j=0}^{d} \gamma_{j}(n) P_{n-j}(x)
$$

with coefficients $\gamma_{j}(n)$ independent of $x$ and $\gamma_{d}(n) \neq 0$. Then by a theorem of P. Maroni [31] there exist $d$ functionals $u_{j}, j=0, \ldots, d-1$ on the space of all polynomials $\mathbb{C}[x]$ such that

$$
\begin{aligned}
& u_{k}\left(P_{n} P_{m}\right)=0, \quad m>n d+k, \quad n \geq 0, \\
& u_{k}\left(P_{n} P_{n d+k}\right) \neq 0, \quad n \geq 0,
\end{aligned}
$$

for each $k \in N_{d+1}:=\{0, \ldots, d-1\}$. When $d=1$ this is the notion of orthogonal polynomials.

In the last 20-30 years there has been much activity in the study of vector orthogonal polynomials and the broader class of multiple orthogonal polynomials (see the cited references below).

Applications of $d$-orthogonal polynomials include the simultaneous Padé approximation problem where the multiple orthogonal polynomials appear [2, 3, 4, 5, 14]. Multiple orthogonal polynomials play an important role in random matrix theory $[4,10]$. Applications to integrable systems and quantum mechanics are found as well $[12,13]$. 
One problem that deserves attention is to find analogs of classical orthogonal polynomials. Several authors $[26,34]$ have found multiple orthogonal polynomials, that share a number of properties with the classical orthogonal polynomials - they have raising and lowering operators, Rodrigues type formulas, Pearson equations for the weights, etc. However one of the features of the classical orthogonal polynomials - a differential operator for which the polynomials are eigenfunction, is missing. Sometimes this property is relaxed to the property that the polynomials satisfy linear differential equation, whose coefficients may depend on the degree of the polynomial.

The ideas from bispectral theory that we use, allow us to to construct new VOP systems, having the strict property to be eigenfunctions of one differential operator. This is even stronger than one of the research problems suggested in [34]. We notice that Hahn's characterization, while valid for the polynomials of Hermite type (Appell polynomials) is not shared by the new VOP, constructed in Sections 4 and 5 (this was guessed in [34]). In these cases the relevant property is a generalization of Sheffer's one: There exist an operator $Q(x, \partial)$ such that

$$
Q(x, \partial) P_{n}(x)=\mu(n) P_{n-1} .
$$

Recall that Sheffer's property corresponds to $\mu(n)=n$ and $Q=\partial$. But in the constructions in the present paper $\mu(n)$ is a polynomial of degree higher than one and $Q$ can be a higher-order differential operator.

We are looking for polynomials $P_{n}(x), n=0,1, \ldots$ that are eigenfunctions of a differential operator $L$ with eigenvalues depending on the discrete variable $n$ (the index) as in (1.1) and which at the same time are eigenfunctions of a difference operator in $n$, i.e., finite-term recursion relation (1.3) with an eigenfunction, depending only on the continuous variable $x$. This is a $(d+2)$-order recursion relation with some $d \geq 1$. Systems with these properties will be called polynomial systems with Bochner's property.

One of our results includes an extension of Laguerre polynomials. We construct systems of vector orthogonal polynomials $\left\{P_{n}(x)\right\}$ which are eigenfunctions of a differential operator starting from a very simple bispectral situation. Our approach uses ideas of the bispectral theory from [6] but does not use Darboux transformations, which is usually the case, see, e.g., [19, 21, 22, 24, 25]. We use methods introduced in [6] which we describe below. Also a well known extension of Hermite polynomials (see [1, 15, 32]) is presented. The reason to repeat this well known result is that our construction is a new one. Also the case of Hermite-like polynomials is the simplest and illustrates the idea without going to computations.

Here is a sketch of the method explained on the example of Hermite-like polynomials. We start with the Weyl algebra $W_{1}$, i.e., the algebra of differential operators in one variable $x$ with polynomial coefficients. Another algebra $\mathcal{R}$ is spanned by the shift operator $T$ in $n$, $T f(n)=f(n+1)$, its inverse $T^{-1}$ and $n$. There is a very simple example of the bispectral problem with $\psi(x, n)=x^{n}$. It is a joint eigenfunction of the operators $H=x \partial_{x}$ and the shift operator $T$. In more details

$$
H \psi(x, n)=n \psi(x, n), \quad T \psi(x, n)=x \psi(x, n) .
$$

This defines an anti-involution $b: W_{1} \rightarrow \mathcal{R}, b(H)=n, b(T)=x, b(\partial)=n T^{-1}$. If we perform an automorphism of the Weyl algebra $\sigma: W_{1} \rightarrow W_{1}$ we can obtain another anti-isomorphism $b^{\prime}=b \circ \sigma^{-1}: W_{1} \rightarrow \mathcal{R}$. This will define a new bispectral problem by taking $H^{\prime}=b \circ \sigma(H)$ and $\Lambda=b \circ \sigma^{-1}(x)$. In the case described briefly here we obtain Hermite polynomials from the simplest nontrivial automorphism $\sigma=e^{\operatorname{ad}_{B}}$ with $B=-\partial^{2} / 2$. Using other automorphisms we obtain systems of vector orthogonal polynomials in general, which are known under the name Appell polynomial systems [1, 15, 32].

Instead of $W_{1}$ we can use other algebras. For example we can take a suitable enveloping algebra of $\mathfrak{s l}_{2}$. This will give us Laguerre polynomials and some of their vector orthogonal 
generalizations. Using a different algebra in Section 5, we come to new classes of VOP. In fact its first member is also known and can be found in [8,9].

In [36] the authors use automorphisms of the Heisenberg-Weyl algebra to construct vector orthogonal polynomials of Hermite and Charlier type. A nice feature of their construction is that a connection to representation theory is underlined and heavily used. Our construction uses only automorphism of algebras as defined in [6] and seems to be simpler and easier to apply elsewhere.

Here we keep the exposition as simple as possible. We do not study properties of the polynomial systems and leave this for future work. The construction of the present paper can be performed in much more general situations of VOP, including their discrete versions, see [23], matrix orthogonal polynomials, multivariable orthogonal polynomials, etc. This will be done elsewhere.

\section{Elements of bispectral theory}

The following introductory material is mainly borrowed from [6]. Here we present it in a form suitable for the continuous-discrete version of the bispectral problem.

For $i=1,2$, let $\Omega_{i}$ be two open subsets of $\mathbb{C}$ such that $\Omega_{2}$ is invariant under the translation operator $T: n \mapsto n+1$ and its inverse $T^{-1}$.

A complex analytic difference operator on $\Omega_{2}$ is a finite sum of the form

$$
\sum_{k \in \mathbb{Z}} c_{k}(n) T^{k}
$$

where $c_{k}: \Omega_{1} \rightarrow \mathbb{C}$ are analytic functions.

By $\mathcal{B}_{1}$ we denote an algebra with unit, consisting of differential operators $L(x, \partial)$ in one variable $x$. By $\mathcal{B}_{2}$ we denote an algebra of difference operators $\Lambda(n, T)$ with unit. Denote by $\mathcal{M}$ the space of complex analytic functions on $\Omega_{1} \times \Omega_{2}$. The space $\mathcal{M}$ is naturally equipped with the structure of bimodule over the algebra of analytic differential operators $L\left(x, \partial_{x}\right)$ on $\Omega_{1}$ and the difference operators on $\Omega_{2}$.

Assume that there exists an algebra isomorphism $b: \mathcal{B}_{1} \rightarrow \mathcal{B}_{2}$ and an element $\psi \in \mathcal{M}$ such that $P \psi=b(P) \psi, \forall P \in \mathcal{B}_{1}$. Assume that $A_{i}$ and $K_{i}$ are subalgebras of $\mathcal{B}_{i}$ such that $b\left(A_{1}\right)=K_{2}$ and $b\left(K_{1}\right)=A_{2}$. In our case $K_{1}$ will be an algebra of polynomials in $x$ and $K_{2}$ will be an algebra of polynomials in $n$.

A continuous-discrete bispectral function is by definition an element of $\mathcal{M}$ (i.e., an analytic function) $\psi: \Omega_{1} \times \Omega_{2} \rightarrow \mathbb{C}$ for which there exist analytic differential operator $L\left(x, \partial_{x}\right)$ on $\Omega_{1}$, analytic difference operator $\Lambda(n, T)$ on $\Omega_{2}$, and analytic functions $\theta(x)$ and $\lambda(n)$, such that

$$
L\left(x, \partial_{x}\right) \psi(x, n)=\lambda(n) \psi(x, n), \quad \Lambda\left(n, T_{n}\right) \psi(x, n)=\theta(x) \psi(x, n)
$$

on $\Omega_{1} \times \Omega_{2}$. In fact, as we would be interested in VOP, we will consider only the case when $\theta(x) \equiv x$. We will assume that $\psi(x, n)$ is a nonsplit function of $x$ and $n$ in the sense that it satisfies the condition

(**) there are no nonzero analytic difference operators $L\left(x, \partial_{x}\right)$ and $\Lambda(n, T)$ that satisfy one of the above conditions with $f(n) \equiv 0$ or $\theta(x) \equiv 0$.

The assumption (**) implies that the map $b: \mathcal{B}_{1} \rightarrow \mathcal{B}_{2}$, given by $b\left(P\left(x, \partial_{x}\right)\right):=S(n, T)$ is a well defined algebra anti-isomorphism. The algebra $A_{1}:=b^{-1}\left(K_{2}\right)$ consists of the bispectral operators corresponding to $\psi(x, z)$ (i.e., differential operators in $x$ having the properties $(2.1)$ ) and the algebra $A_{2}:=b\left(K_{1}\right)$ consists of the bispectral operators corresponding to $\psi(x, n)$ (i.e., difference operators in $n$ having the properties (2.1)). 
Below we present the differential-difference version of the general bispectral problem, suitable in the set-up of vector orthogonal polynomial sequences, which are eigenfunctions of differential operators. We are interested in the case when, for any fixed $n$, the function $\psi(x, n)$ defining the map $b$ is a polynomial in $x$. We additionally assume that, for all $n$, polynomials $\psi(x, n)$ are the eigenfunctions of a fixed differential operator in the variable $x$ and that, for any fixed $x$, the function $\psi(x, n)$ is an eigenfunction of a difference operator in $n$. We know that such a situation occurs in case of the classical orthogonal polynomials.

Let $\mathcal{B}_{1}$ be the Weyl algebra $W_{1}$ (spanned over $\mathbb{C}$ by $x$ and $\partial$ ). To define another algebra, we introduce three operators: the shift operator $T$ acting on functions $f(n)$ of the discrete variable $n$ by $T f(n):=f(n+1)$, its inverse $T^{-1}$ shifting in the opposite direction, i.e., $T^{-1} f(n):=f(n-1)$, and the operator $n$ of multiplication by the variable $n$. We define the algebra $\mathcal{R}_{2}$ of difference operators over $\mathbb{C}$, spanned by $T, T^{-1}$ and $n$ with the obvious relations. Let $\mathcal{M}$ be a left module over $\mathcal{B}_{1}$ and $\mathcal{R}_{2}$. In our case such a module is a linear space of bivariate analytic functions $f(x, n)$, where $x$ is a continuous and $n$ is a discrete variables.

Set $L:=x \partial$. Introducing $\psi(x, n):=S_{n}(x):=x^{n}$, we get

$$
\begin{aligned}
& L S_{n}(x)=n S_{n}(x), \\
& x S_{n}(x)=S_{n+1}(x), \\
& \partial S_{n}(x)=n S_{n-1}(x) .
\end{aligned}
$$

We see that (2.2) and (2.3) are providing an instance of a differential-difference bispectral pair of operators. The equation (2.2) guarantees that $\psi(x, n)$ is an eigenfunction of the differential operator $L$, and (2.3) shows that the same function is an eigenfunction of the difference operator $T$. Equation (2.4) follows from the previous two; it is called the differentiation (or the lowering) formula and is quite important.

We can define the map $b: \mathcal{B}_{1} \rightarrow \mathcal{R}_{2}$ as given by

$$
b(\partial)=n T^{-1}, \quad b(x)=T, \quad b(L)=n .
$$

(It is clear that the first two identities are sufficient to determine $b$ completely. The last identity is included because of its importance and convenience.) Then we put $\mathcal{B}_{2}=b\left(\mathcal{B}_{1}\right)$.

Next we will discuss how to construct new bispectral operators from already known ones. First recall that for an operator $L$ it is said that $\operatorname{ad}_{L}$ acts locally nilpotently when for any element $a \in \mathcal{B}$ there exists $k \in \mathbb{N}$, such that $\operatorname{ad}_{L}^{k}(a)=0$.

The main tool for constructing bispectral operators in this paper will be the following simple observation made in [6].

Proposition 2.1. Let $\mathcal{B}_{1}, \mathcal{B}_{2}$ be unital algebras with the properties described above and $b: \mathcal{B}_{1} \rightarrow \mathcal{B}_{2}$. Let $L \in \mathcal{B}_{1}$ and $\operatorname{ad}_{L}: \mathcal{B}_{1} \rightarrow \mathcal{B}_{1}$ be a locally nilpotent operator. Suppose that, for any fixed $n, e^{L} \psi(x, n)$ is a polynomial in $x$ of degree $n$. Define a new map $b^{\prime}: \mathcal{B}_{1} \rightarrow \mathcal{B}_{2}$ via the new polynomial function $\psi^{\prime}(x, n):=e^{L} \psi(x, n)$. Then $b^{\prime}=b\left(e^{-\operatorname{ad}_{L}}\right)$ and $b^{\prime}: \mathcal{B}_{1} \rightarrow \mathcal{B}_{2}$ is a bispectral anti-involution.

\section{$3 \quad$ Hermite-like polynomials}

Observe that $\operatorname{ad}_{\partial}$ acts locally nilpotently on the algebra $\mathcal{B}_{1}$. The same is true when we take instead of $\partial$ any polynomial $q(\partial)$ without a free term. From this it follows that for any element $a \in \mathcal{B}_{1}$, the series

$$
\sum_{k=0}^{\infty} \frac{\operatorname{ad}_{\partial}^{k}(a)}{k !}
$$


has only finitely many terms. For such a polynomial $q(\partial)$ we define the automorphism $\sigma:=e^{\operatorname{ad}_{q(\partial)}}$. Its action on $L$ is explicitly given by

$$
\sigma(L)=L+\sum_{k=1}^{\infty} \frac{\operatorname{ad}_{q(\partial)}^{k}(L)}{k !}=L+[q(\partial), L]=L+q^{\prime}(\partial) \partial,
$$

since the rest of the terms vanish.

Let us first compute the action of $\sigma=e^{\operatorname{ad}_{q(\partial)}}$ on the standard basis of $\mathcal{B}_{1}$. We have

$$
\sigma(\partial)=\partial, \quad \sigma(x)=x+q^{\prime}(\partial) .
$$

Next we define a new map $b^{\prime}: \mathcal{B}_{1} \rightarrow \mathcal{B}_{2}$ by $b^{\prime}:=b \circ \sigma^{-1}$. We will introduce the new function $\psi_{1}(x, n)$ defined via

$$
\psi_{1}(x, n):=P_{n}^{q}(x):=e^{q(\partial)} x^{n}=x^{n}+\sum_{m=1}^{\infty} \frac{(q(\partial))^{m} x^{n}}{m !} .
$$

Due to the fact that $q(\partial)$ reduces the degrees of the polynomials it is clear that the latter series contains only a finite number of terms. Hence it is a polynomial in $x$. (Here we use that $q$ has no constant term.) Define the operator $L_{1}:=\sigma(L)$. We already saw that

$$
L_{1}=x \partial+q^{\prime}(\partial) \partial \text {. }
$$

Lemma 3.1. The new bispectral anti-involution $b^{\prime}$ satisfies:

$$
b^{\prime}(\partial)=n T^{-1}, \quad b^{\prime}(x)=T-q^{\prime}\left(n T^{-1}\right), \quad b^{\prime}\left(L_{1}\right)=n .
$$

Proof. We have $b^{\prime}(\partial)=b \circ \sigma^{-1}(\partial)=b(\partial)=n T^{-1}$. In the same way we compute $b^{\prime}(x)=$ $b \circ \sigma^{-1}(x)=b\left(x-q^{\prime}(\partial)\right)=T-q^{\prime}\left(n T^{-1}\right)$. Finally for $L_{1}$ we find $b^{\prime}\left(L_{1}\right)=b \circ \sigma^{-1}\left(L_{1}\right)=$ $b \circ \sigma^{-1} \circ \sigma(L)=b(L)=n$.

Theorem 3.2. The polynomials $P_{n}^{q}$ have the following properties:

(i) they are eigenfunctions of the differential operator

$$
L_{1}:=q^{\prime}(\partial) \partial+x \partial
$$

(ii) they satisfy the recurrence relation

$$
x P_{n}^{q}(x)=P_{n+1}^{q}-q^{\prime}\left(n T^{-1}\right) P_{n}^{q}(x) ;
$$

(iii) the following differentiation formula (lowering operator)

$$
\partial P_{n}^{q}(x)=n P_{n-1}^{q}
$$

holds.

Proof. Indeed, statement (i) that polynomials $P_{n}(x)$ are eigenfunctions of the operator

$$
L_{1}=q^{\prime}(\partial) \partial+x \partial
$$

follows from the computation of the new bispectral involution $b^{\prime}$ in Lemma 3.1. More exactly, the third equation of (3.1) gives

$$
\left(q^{\prime}(\partial) \partial+x \partial\right) P_{n}(x)=n P_{n}(x) .
$$

To prove recurrence (ii), observe the second equation in (3.1) provides

$$
x P_{n}(x)=P_{n+1}-q^{\prime}\left(n T^{-1}\right) P_{n} .
$$

Alsothe first equation in (3.1) gives the differentiation formula $\partial P_{n}=n P_{n-1}$, which settles (iii). 


\section{Laguerre-like VOP}

We start with an algebra $\mathcal{B}_{1}$ spanned by the operators $x, H=x \partial$ and $B=x \partial^{2}+\beta \partial$. The commutation relations are

$$
[H, x]=x, \quad[B, x]=2 H+\beta, \quad[H, B]=-B .
$$

This shows that our algebra is an enveloping algebra of $\mathfrak{s l}_{2}$ (not the universal one).

Next we define the algebra $\mathcal{R}_{2}$ of difference operators over $\mathbb{C}$, as the one spanned by $T, T^{-1}, n$. Let $\mathcal{M}$ be a left module over $\mathcal{B}_{1}$ and $\mathcal{R}_{2}$. In our case such a module is a linear space of bivariate functions $f(x, n)$, where $x$ is a continuous and $n$ is a discrete variables. We define $K_{1}$ to be the algebra of polynomials in $x$ and $K_{2}$ to be the algebra of polynomials in $n$.

Let again $\psi(x, n):=S_{n}(X):=x^{n}$. It is obviously nonsplit in the above defined sense $(* *)$. Define the map $b$ by its action on the generators:

$$
b(x)=T, \quad b(H)=n, \quad b(B)=n(n-1+\beta) T^{-1} .
$$

We define the algebra $\mathcal{B}_{2}$ to be the image $b\left(\mathcal{B}_{1}\right)$ of $\mathcal{B}_{1}$ under the map $b$. Obviously the map $\sigma: \mathcal{B}_{1} \rightarrow \mathcal{B}_{2}$ is an anti-automorphism.

Setting $L:=H$ we get

$$
H S_{n}(x)=n S_{n}(x), \quad x S_{n}(x)=S_{n+1}(x), \quad B S_{n}(x)=n(n-1+\beta) S_{n-1}(x) .
$$

Using the above notation and following the scheme of the previous section, we now set up an appropriate bispectral problem. We will use the algebras $\mathcal{B}_{1}$ and $\mathcal{B}_{2}$ introduced there.

Consider a polynomial $q(X)$. For simplicity we assume that $q(X)$ has no constant term. Let us define an automorphism $\sigma: \mathcal{B}_{1} \rightarrow \mathcal{B}_{1}$

$$
\sigma(A)=e^{\operatorname{ad}_{q(B)}(A), \quad A \in \mathcal{B}_{1} .}
$$

In the next lemma we compute the action of $\sigma$.

Lemma 4.1. $\sigma$ acts on the generators as follows

$$
\begin{aligned}
& \sigma(x)=x+(2 H+\beta) q^{\prime}(B)+q^{\prime \prime}(B) B+q^{\prime}(B)^{2} B, \\
& \sigma(H)=H+q^{\prime}(B) B, \quad \sigma(B)=B .
\end{aligned}
$$

Proof. In order to derive the first formula we need to compute the commutator

$$
\left[B^{m}, x\right]=2 \sum_{j=0}^{m-1} B^{j} H B^{m-1-j}+\beta B^{m-1} .
$$

Notice that $B^{j} H=(H+j) B^{j}$. From this we find

$$
\left[B^{m}, x\right]=2 m H B^{m-1}+(m(m-1)+\beta m) B^{m-1},
$$

which shows that

$$
[q(B), x]=(2 H+\beta) q^{\prime}(B)+q^{\prime \prime}(B) B .
$$

In order to compute $\operatorname{ad}_{q}^{2}(x)$ we also need $[q(B), H]=q^{\prime}(B) B$, which yields

$$
\left[q(B), H q^{\prime}(B)\right]=q^{\prime 2}(B) B .
$$

This gives that

$$
\operatorname{ad}_{q}^{2}(x)=2 q^{\prime 2}(B) B, \quad \operatorname{ad}_{q}^{3}(x)=0 .
$$

The two other formulas are obvious. 
The lemma shows that $\operatorname{ad}_{q(B)}$ acts locally nilpotently on the algebra $\mathcal{B}_{1}$, i.e., when applied to any element $A \in \mathcal{B}_{1}$, the series

$$
\sum_{k=0}^{\infty} \frac{\operatorname{ad}_{q(B)}^{k}(A)}{k !}
$$

has only finitely many terms.

Let us define a new map $b^{\prime}: \mathcal{B}_{1} \rightarrow \mathcal{B}_{2}$ given by $b^{\prime}:=b \circ \sigma^{-1}$ and a new function $\psi_{1}(x, n)$ defined via

$$
\psi_{1}(x, n):=P_{n}^{q}(x):=e^{q(B)} x^{n}=x^{n}+\sum_{k=1}^{\infty} \frac{(q(B))^{k} x^{n}}{k !} .
$$

It is clear that the latter series contains only a finite number of terms as the operator $q(B)$ reduces the degrees of the polynomials. (Here we use that $q$ has no constant term.) Hence it is a polynomial in $x$.

Below and further in the paper we also use without mentioning that

$$
\sigma^{-1}=\sum_{j=0}^{\infty} \frac{(-\mathrm{ad})_{q(B)}^{j}}{j !} .
$$

Define the operator $L_{1}:=\sigma(L)$. One can easily see that $L_{1}=x \partial+q^{\prime}(B) B$.

Lemma 4.2. The new bispectral involution $b^{\prime}$ on $\mathcal{B}^{\prime}$ satisfies:

$$
\begin{aligned}
& b^{\prime}(B)=n(n-1+\beta) T^{-1}, \\
& b^{\prime}(x)=T-q^{\prime}(b(B))(2 n+\beta)-q^{\prime \prime}(b(B)) b(B)+q^{\prime 2}(b(B)) b(B), \\
& b^{\prime}\left(L_{1}\right)=n .
\end{aligned}
$$

Proof. We have $b^{\prime}(B)=b \circ \sigma^{-1}(B)=b(B)=n(n-1+\beta) T^{-1}$. In the same way we compute

$$
\begin{aligned}
b^{\prime}(x) & =b \circ \sigma^{-1}(x)=b\left(x-(2 H+\beta) q^{\prime}(B)-q^{\prime \prime}(B) B+q^{\prime 2}(B) B\right) \\
& =T-q^{\prime}(b(B))(2 n+\beta)-q^{\prime \prime}(b(B)) b(B)+q^{\prime 2}(b(B)) b(B) .
\end{aligned}
$$

Finally for $L_{1}$ we find $b^{\prime}\left(L_{1}\right)=b \circ \sigma^{-1}\left(L_{1}\right)=b \circ \sigma^{-1} \circ \sigma(L)=b(L)=n$.

Theorem 4.3. The polynomials $P_{n}^{q}$ have the following properties:

(i) they are eigenfunctions of the differential operator

$$
L_{1}:=q^{\prime}(B) B+x \partial ;
$$

(ii) they satisfy the recurrence relation

$$
\begin{aligned}
x P_{n}^{q}(x)= & P_{n+1}^{q}-q^{\prime}\left(n(n-1+\beta) T^{-1}\right)(2 n+\beta) P_{n}^{q}(x) \\
& +\left\{-2 q^{\prime \prime}\left(n(n-1+\beta) T^{-1}\right)+q^{\prime 2}\left(n(n-1+\beta) T^{-1}\right)\right\} n(n-1+\beta) P_{n-1}^{q}(x) ;
\end{aligned}
$$

(iii) the following differentiation formula (lowering operator)

$$
B P_{n}^{q}(x)=n(n-1+\beta) P_{n-1}^{q}
$$

holds.

Proof. All the statements follow easily from the computation of the new bispectral involution $b^{\prime}$ in Lemma 4.2. Essentially the proof is the same as the proof for Hermite-like polynomials. 


\section{Automorphisms generated by third-order operators}

We start with an algebra $\mathcal{B}_{1}$ spanned by the operators $x, H=x \partial$ and $B=x^{2} \partial^{3}+\alpha x \partial^{2}+\beta \partial$. The commutation relations are

$$
[H, x]=x, \quad[B, x]=W:=3 H^{2}+2 \alpha H+\beta, \quad[H, B]=-B .
$$

We again use the algebra $\mathcal{R}_{2}$ of difference operators over $\mathbb{C}$, spanned by $T, T^{-1}, n$ and the bimodule $\mathcal{M}$ of bivariate functions $f(x, n)$, where $x$ is a continuous and $n$ is a discrete variables.

Let $S_{n}(x):=\psi(x, n):=x^{n}$. It is obviously nonsplit in the above defined sense (**). Define the map $b: \mathcal{B}_{1} \rightarrow \mathcal{R}_{2}$ by its action on the generators:

$$
b(x)=T, \quad b(H)=n, \quad b(B)=n[(n-1)(n-2)+\alpha(n-1)+\beta] T^{-1} .
$$

The algebra $\mathcal{B}_{2}$ is the image of $\mathcal{B}_{1}$ under the map $b$.

Set $L:=H$. Then

$$
\begin{aligned}
& H S_{n}(x)=n S_{n}(x) \\
& x S_{n}(x)=S_{n+1}(x) \\
& B S_{n}(x)=\left[\left(\begin{array}{l}
n \\
3
\end{array}\right)+\alpha\left(\begin{array}{l}
n \\
2
\end{array}\right)+\beta n\right] S_{n-1}(x) .
\end{aligned}
$$

We see that (5.1) and (5.2) are providing an instance of a differential-difference bispectral pair of operators. Namely, we get that (5.1) guarantees that $\psi(x, n)$ is an eigenfunction of the differential operator $L$, and (5.2) shows that the same function is an eigenfunction of the difference operator $T$.

Using the above notation and following the scheme of Section 3, we now set up an appropriate bispectral problem.

Consider a polynomial $q(X)$ and for simplicity we assume that $q(X)$ has no constant term. Define an automorphism of the algebra $\mathcal{B}_{1}$ :

$$
\sigma(A)=e^{\operatorname{ad}_{q(B)}(A), \quad A \in \mathcal{B}_{1} .}
$$

Let us compute the action of $\sigma$. Put $R=3(2 H+1)+2 \alpha$.

Lemma 5.1. $\sigma$ acts on the generators as follows

$$
\begin{aligned}
\sigma(x)= & x+W q^{\prime}(B)+R q^{\prime \prime}(B) B / 2+q^{\prime \prime \prime}(B) B^{2} \\
& +\left\{3 q^{\prime \prime}(B) B+(R+6) q^{\prime}(B) / 2+q^{\prime 2}(B) B\right\} B q^{\prime}(B), \\
\sigma(H) & =H+q^{\prime}(B) B, \quad \sigma(B)=B .
\end{aligned}
$$

Proof. To prove the first identity we will need to compute the commutator $\left[B^{m}, x\right]$. First we have

$$
\left[B^{m}, x\right]=\sum_{j=0}^{m-1} B^{j} W B^{m-1-j}
$$

Notice that $B W=W B+R B$. We also need $B^{j} R=(R+6 j) B^{j}$, from which we find

$$
B^{j} W=W B^{j}+\sum_{k=0}^{j-1} B^{k} R B^{j-k}=\left(W+\sum_{k=0}^{j-1}(R+6 k)\right) B^{j}=[W+j R+3 j(j-1)] B^{j} .
$$


Hence

$$
\left[B^{m}, x\right]=\sum_{j=0}^{m-1}[W+j R+3 j(j-1)] B^{m-1} .
$$

Then using the formulas for sums of powers we transform this expression into

$$
\left[B^{m}, x\right]=[m W+m(m-1) R / 2+m(m-1)(m-2)] B^{m-1},
$$

which shows that

$$
[q(B), x]=W q^{\prime}(B)+R q^{\prime \prime}(B) B / 2+q^{\prime \prime \prime}(B) B^{2} .
$$

Using the commutation relation $[B, H]=B$ we compute

$$
[q(B), H]=q^{\prime}(B) B
$$

and

$$
[q(B), W]=3 / 2 q^{\prime \prime}(B) B^{2}+R q^{\prime}(B) B .
$$

This gives that

$$
\operatorname{ad}_{q}^{2}(x)=\left\{6 q^{\prime \prime}(B) B+(R+6) q^{\prime}(B)\right\} B q^{\prime}(B) .
$$

Finally we find

$$
\operatorname{ad}_{q}^{3}(x)=6 q^{\prime 3}(B) B^{2}, \quad \operatorname{ad}_{q}^{4}(x)=0 .
$$

This gives the first identity. The other two are obvious.

The lemma shows that $\operatorname{ad}_{q(B)}$ acts locally nilpotently on the algebra $\mathcal{B}$, i.e., when applied to any element $A \in \mathcal{B}$, the series

$$
\sum_{k=0}^{\infty} \frac{\operatorname{ad}_{q(B)}^{k}(A)}{k !}
$$

has only finite number of terms.

Let us define a new map $b^{\prime}: \mathcal{B}_{1} \rightarrow \mathcal{B}_{2}$ given by $b^{\prime}:=b \circ \sigma^{-1}$ and the new function $\psi_{1}(x, n)$ defined via

$$
\psi_{1}(x, n):=P_{n}^{q}(x):=e^{q(B)} x^{n}=x^{n}+\sum_{k=1}^{\infty} \frac{q^{k}(B) x^{n}}{k !} .
$$

It is clear that the latter series contains only a finite number of terms as the operator $q(B)$ reduces the degrees of the polynomials. Hence $\psi_{1}(x, n)$ is a polynomial in $x$. (Here we use that $q$ has no constant term.) Define the operator $L_{1}:=\sigma(L)$. One can easily see that

$$
L_{1}=x \partial+q^{\prime}(B) B
$$

In order to simplify the notations let us put

$$
\begin{aligned}
& Q_{0}(B)=-q^{\prime \prime \prime}(B) B^{2}+\left\{2 q^{\prime \prime}(B) B+3 q^{\prime}(B)-q^{2}(B) B\right\} B q^{\prime}(B), \\
& Q_{1}(B)=\frac{\left(q^{\prime 2}(B)-q^{\prime \prime}(B)\right) B}{2} .
\end{aligned}
$$


Lemma 5.2. The new bispectral anti-involution $b^{\prime}$ satisfies:

$$
\begin{aligned}
& b^{\prime}(B)=n[(n-1)(n-2)+\alpha(n-1)+\beta] T^{-1}, \\
& b^{\prime}(x)=T-q^{\prime}(b(B))\left(3 n^{2}+2 \alpha n+\beta\right)+Q_{1}(b(B))(6 n+3+\alpha)+Q_{0}(b(B)), \\
& b^{\prime}\left(L_{1}\right)=n .
\end{aligned}
$$

Proof. Repeats the proof of Lemma 4.2 and will be omitted.

We formulate the main results about the polynomial system $P_{n}^{q}(x)$ in the next theorem.

Theorem 5.3. The polynomials $P_{n}^{q}(x)$ have the following properties:

(i) they are eigenfunctions of the differential operator

$$
L_{1}:=q^{\prime}(B) B+x \partial
$$

(ii) they satisfy the recursion relation

$$
\begin{aligned}
x P_{n}^{q}(x)= & P_{n+1}^{q}+\left\{-3 q^{\prime}(b(B))\left(3 n^{2}+2 \alpha n+\beta\right)+Q_{1}(b(B))(6 n+3+\alpha)\right. \\
& \left.+Q_{0}(b(B))\right\} P_{n}^{q}(x)
\end{aligned}
$$

(iii) the following differentiation formula (lowering operator)

$$
B P_{n}^{q}(x)=n[(n-1)(n-2)+\alpha(n-1)+\beta] P_{n-1}^{q}
$$

holds.

Proof repeats that of Theorem 3.2.

\section{Examples}

In the examples below we construct the differential operator $L_{1}$ that has the vector orthogonal sets $P_{n}^{q}(x)=e^{q(B)} x^{n}$ as eigenfunctions. In some cases we also write explicitly the finite-term recursion relation.

Example 6.1. Set $q(\partial)=-1 / k \partial^{k}$. Then we obtain

$$
L_{1}=x \partial-\partial^{k} .
$$

Observe that, for $k=2$, the operator $-L_{1}$ is the standard Hermite operator. For $k>2$, $-L_{1}$ appeared earlier in [33]. The recurrence relation for $P_{n}(x)$ is given by (3.3). In the present case it reads

$$
x P_{n}(x)=P_{n+1}(x)+\frac{n !}{(k-1) !} P_{n-k+1}(x),
$$

which agrees with the results in [33]. In general all the examples of Section 3 have been studied thoroughly starting with the classical paper [1]. In the context of vector orthogonal polynomials the results have been found in [15].

Example 6.2. Here we take $B=x \partial^{2}+\beta \partial$. Let us take the polynomial $q(B)=B$. In this case we have

$$
L_{1}=H+q^{\prime}(B) B=x \partial^{2}+\beta \partial+x \partial,
$$

which up to change of the variables is the Laguerre operator. 
Example 6.3. The simplest new example is given by the polynomial $q(B)=B^{2} / 2, B=$ $x \partial^{2}+\beta \partial$. In this case we have

$$
L_{1}=H+q^{\prime}(B) B=\left(x \partial^{2}+\beta \partial\right)^{2}+x \partial=x^{2} \partial^{4}+2 x(1+\beta) \partial^{3}+\left(\beta+\beta^{2}\right) \partial^{2}+x \partial .
$$

The 5-terms recurrence relation reads

$$
x P_{n}(x)=P_{n+1}-n(n-1+\beta)(2 n-1+\beta) P_{n-1}+\frac{n !}{3 !}\left(\begin{array}{c}
n-1+\beta \\
3
\end{array}\right) P_{n-3} .
$$

Here are the first few polynomials

$$
\begin{aligned}
& P_{0}(x)=1, \quad P_{1}(x)=x, \quad P_{2}(x)=x^{2}+\beta(1+\beta), \\
& P_{3}(x)=x^{3}+3(1+\beta)(2+\beta) x, \quad \ldots
\end{aligned}
$$

Example 6.4. Let us take the polynomial $q(B)=B, B=x^{2} \partial^{3}+\alpha x \partial^{2}+\beta \partial$. Then

$$
L_{1}=H+q^{\prime}(B) B=x^{2} \partial^{3}+\alpha x \partial^{2}+\beta \partial+x \partial .
$$

The corresponding polynomials satisfy the 4 -term recurrence relation

$$
\begin{aligned}
x P_{n}^{q}(x)= & P_{n+1}^{q}+(3-2 \alpha) P_{n}^{q}(x)-3 n[(n-1)(n-2+\alpha)+\beta] P_{n-1}^{q} \\
& -n(n-1)[(n-1)(n-2+\alpha)+\beta][(n-2)(n-3+\alpha)+\beta] P_{n-2}^{q} .
\end{aligned}
$$

This example has been produced in [7] within the context of vector orthogonal polynomials which are generalized hypergeometric functions.

Example 6.5. Let us take the polynomial $q(B)=B^{2} / 2, B=x^{2} \partial^{3}$. Then

$$
L_{1}=H+q^{\prime}(B) B=\left(x^{2} \partial^{3}\right)^{2}+x \partial=x^{4} \partial^{6}+6 x^{3} \partial^{5}+6 x^{2} \partial^{4}+x \partial .
$$

This example seems to be new as most of the examples in Sections 4 and 5 .

The polynomials satisfy a 7-term recurrence relation which we skip as it is not much simpler than the corresponding general formula (5.3). Here are the first few polynomials:

$$
\begin{aligned}
& P_{0}(x)=1, \quad P_{1}(x)=x, \quad P_{2}(x)=x^{2}, \\
& P_{3}(x)=x^{3}+3 \cdot 2^{3} x, \quad P_{4}(x)=x^{4}+4 \cdot 3^{3} \cdot 2^{2} x^{2}, \quad \ldots
\end{aligned}
$$

\section{Acknowledgements}

The author is deeply grateful to Boris Shapiro for showing and discussing some examples of systems of polynomials studied here and in particular the examples from [33]. Without this probably the project would have never be even started. Also Plamen Iliev made valuable comments on some results generalizing Laguerre polynomials, which helped me to place my work among the rest of the research. Milen Yakimov pointed out several errors. Many thanks go to the referees and the editor who pointed out a number of incorrect formulas and misprints and thus helped me to improve considerably the initial text. The author is grateful to the Mathematics Department of Stockholm University for the hospitality in April 2015. Last but not least I am extremely grateful to Professors T. Tanev and K. Kostadinov, and Mrs. Z. Karova from the Bulgarian Ministry of Education and Science and Professor P. Dolashka, who helped me in the difficult situation when I was sacked by Sofia university in violations of the Bulgarian laws ${ }^{2}$.

\footnotetext{
${ }^{2}$ See EMS Newsletter, p. 71: http://www.ems-ph.org/journals/newsletter/pdf/2015-12-98.pdf.
} 


\section{References}

[1] Appell P., Sur une classe de polynômes, Ann. Sci. École Norm. Sup. (2) 9 (1880), 119-144.

[2] Aptekarev A.I., Multiple orthogonal polynomials, J. Comput. Appl. Math. 99 (1998), 423-447.

[3] Aptekarev A.I., Branquinho A., Van Assche W., Multiple orthogonal polynomials for classical weights, Trans. Amer. Math. Soc. 355 (2003), 3887-3914.

[4] Aptekarev A.I., Kuijlaars A.B.J., Hermite-Padé approximations and ensembles of multiple orthogonal polynomials, Russ. Math. Surv. 66 (2011), 1133-1199.

[5] Aptekarev A.I., Marcellán F., Rocha I.A., Semiclassical multiple orthogonal polynomials and the properties of Jacobi-Bessel polynomials, J. Approx. Theory 90 (1997), 117-146.

[6] Bakalov B., Horozov E., Yakimov M., General methods for constructing bispectral operators, Phys. Lett. A 222 (1996), 59-66, q-alg/9605011.

[7] Ben Cheikh Y., Douak K., A generalized hypergeometric $d$-orthogonal polynomial set, C. R. Acad. Sci. Paris Sér. I Math. 331 (2000), 349-354.

[8] Ben Cheikh Y., Douak K., On the classical $d$-orthogonal polynomials defined by certain generating functions. II, Bull. Belg. Math. Soc. Simon Stevin 8 (2001), 591-605.

[9] Ben Cheikh Y., Ouni A., Some generalized hypergeometric d-orthogonal polynomial sets, J. Math. Anal. Appl. 343 (2008), 464-478.

[10] Bleher P., Delvaux S., Kuijlaars A.B.J., Random matrix model with external source and a constrained vector equilibrium problem, Comm. Pure Appl. Math. 64 (2011), 116-160, arXiv:1001.1238.

[11] Bochner S., Über Sturm-Liouvillesche Polynomsysteme, Math. Z. 29 (1929), 730-736.

[12] Chang J.-H., The Gould-Hopper polynomials in the Novikov-Veselov equation, J. Math. Phys. 52 (2011), 092703, 15 pages, arXiv:1011.1614.

[13] Dattoli G., Lorenzutta S., Maino G., Torre A., Cesarano C., Generalized Hermite polynomials and superGaussian forms, J. Math. Anal. Appl. 203 (1996), 597-609.

[14] de Bruin M.G., Simultaneous Padé approximation and orthogonality, in Polynômes Orthogonaux et Applications, Lecture Notes in Math., Vol. 1171, Editors C. Brezinski, A. Draux, A.P. Magnus, P. Maroni, A. Ronveaux, Springer-Verlag, Berlin, 1985, 74-83.

[15] Douak K., The relation of the $d$-orthogonal polynomials to the Appell polynomials, J. Comput. Appl. Math. 70 (1996), 279-295.

[16] Duistermaat J.J., Grünbaum F.A., Differential equations in the spectral parameter, Comm. Math. Phys. 103 (1986), 177-240.

[17] Durán A.J., de la Iglesia M.D., Differential equations for discrete Laguerre-Sobolev orthogonal polynomials, J. Approx. Theory 195 (2015), 70-88, arXiv:1309.6259.

[18] Grünbaum F.A., Haine L., The q-version of a theorem of Bochner, J. Comput. Appl. Math. 68 (1996), 103-114.

[19] Grünbaum F.A., Haine L., Bispectral Darboux transformations: an extension of the Krall polynomials, Int. Math. Res. Not. (1997), 359-392.

[20] Grünbaum F.A., Haine L., A theorem of Bochner, revisited, in Algebraic Aspects of Integrable Systems, Progr. Nonlinear Differential Equations Appl., Vol. 26, Editors I.M. Gel'fand, T. Fokas, Birkhäuser Boston, Boston, MA, 1997, 143-172.

[21] Grünbaum F.A., Haine L., Horozov E., Some functions that generalize the Krall-Laguerre polynomials, J. Comput. Appl. Math. 106 (1999), 271-297.

[22] Grünbaum F.A., Yakimov M., Discrete bispectral Darboux transformations from Jacobi operators, Pacific J. Math. 204 (2002), 395-431, math.CA/0012191.

[23] Horozov E., Automorphisms of algebras and Bochner's property for discrete vector orthogonal polynomials, arXiv:1602.04343.

[24] Iliev P., Krall-Jacobi commutative algebras of partial differential operators, J. Math. Pures Appl. 96 (2011), 446-461, arXiv:1011.2599.

[25] Iliev P., Krall-Laguerre commutative algebras of ordinary differential operators, Ann. Mat. Pura Appl. 192 (2013), 203-224, arXiv:1107.3773. 
[26] Kaliaguine V., Ronveaux A., On a system of "classical" polynomials of simultaneous orthogonality, J. Comput. Appl. Math. 67 (1996), 207-217.

[27] Koekoek J., Koekoek R., On a differential equation for Koornwinder's generalized Laguerre polynomials, Proc. Amer. Math. Soc. 112 (1991), 1045-1054.

[28] Koornwinder T.H., Orthogonal polynomials with weight function $(1-x)^{\alpha}(1+x)^{\beta}+M \delta(x+1)+N \delta(x-1)$, Canad. Math. Bull. 27 (1984), 205-214.

[29] Krall H.L., On orthogonal polynomials satisfying a certain fourth order differential equation, Pennsylvania State College Studies (1940), no. 6, 24 pages.

[30] Littlejohn L.L., The Krall polynomials: a new class of orthogonal polynomials, Quaest. Math. 5 (1982), 255-265.

[31] Maroni P., L'orthogonalité et les récurrences de polynômes d'ordre supérieur à deux, Ann. Fac. Sci. Toulouse Math. 10 (1989), 105-139.

[32] Sheffer I.M., A differential equation for Appell polynomials, Bull. Amer. Math. Soc. 41 (1935), 914-923.

[33] Tater M., Shapiro B., Asymptotic zero distribution of polynomial solutions to degenerate exactly-solvable equations, Talk at CRM-ICMAT Workshop on Exceptional Orthogonal Polynomials and Exact Solutions in Mathematical Physics (Segovia, 2014), private communication.

[34] Van Assche W., Coussement E., Some classical multiple orthogonal polynomials, J. Comput. Appl. Math. 127 (2001), 317-347, math.CA/0103131.

[35] Van Iseghem J., Vector orthogonal relations. Vector QD-algorithm, J. Comput. Appl. Math. 19 (1987), 141-150.

[36] Vinet L., Zhedanov A., Automorphisms of the Heisenberg-Weyl algebra and $d$-orthogonal polynomials, J. Math. Phys. 50 (2009), 033511, 19 pages. 